\title{
INTERVIEW
}

For reprint orders, please contact: reprints@futuremedicine.com

\section{Breast cancer clinical trials: current issues and possible solutions}

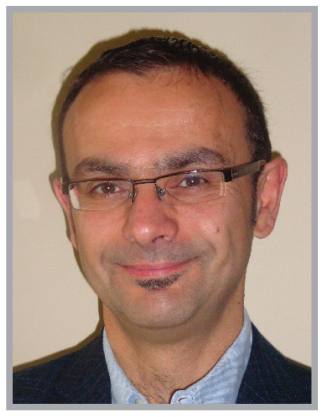

George Dranitsaris* speaks to Alisa Crisp, Commissioning Editor: George Dranitsaris is a health services research consultant with graduate training in biostatistics, pharmacoeconomics, decision analysis and clinical epidemiology. His areas of interest include comparative effectiveness analysis, the measurement of cost-effective drug use in the oncology setting, valuebased drug pricing and the evaluation of drug performance outside the trial setting. He has over 100 publications in the national and international literature, is past President of the Canadian Association of Pharmacy in Oncology, a statistical reviewer for the Journal of Clinical Oncology and a member of the editorial board of the Journal of Oncology Pharmacy Practice and the European Journal of Hospital Pharmacy Science.

Q As a biostatistician, how did you get into the breast cancer field?

Early in my career, when I finished graduate school, I started working at the largest cancer center in Canada, Princess Margaret Hospital, and in that role I interacted in many different disease sites. It just so happened that when I started my career in 1993, Taxol ${ }^{\circledR}$ (Bristol-Myers Squibb, NY, USA) was about to be launched. At the time, Taxol was the first really expensive drug - I think the cost per cycle was something like US\$1500. Back in 1993, that was at least three- or four-fold higher than anything else that we had, because at the time most cancer drugs were US\$200-300 per cycle. That was what introduced me to breast cancer, the fact that this new drug came up, it was very expensive and we had to work out how we could pay for it. I also became interested in pharmacoeconomics, because many pharmacoeconomic issues came up with Taxol. Twenty years later, Taxol is still here, so it is a testament to its effectiveness.

Q What is different about breast cancer in comparison with the other cancers you work on?
With respect to clinical differences, breast cancer is really not one disease but consists of many different subgroups, and this makes it very difficult to treat. We naively put breast cancer into groups that we think are homogeneous, but triplenegative breast cancer, for example, is not a homogeneous group. So that is one difference, the complexity of breast cancer. Clinically, because there are a lot of drugs, relatively speaking, in breast cancer compared with other disease sites, breast cancer patients live for quite a long time. For example, stage 4 breast cancer patients can live 3-4 years, and that makes it difficult with respect to testing new compounds. First, it makes it very difficult to identify a survival difference, especially in early lines of therapy, because of crossover and contamination with competing therapies after the patient progresses from the initial treatment. Second, it becomes very difficult to demonstrate quality-of-life differences, at least the way we currently measure quality of life, because we have all of these competing interventions coming in. At the end of the day, as a very well-respected oncologist told me early in my career, there are

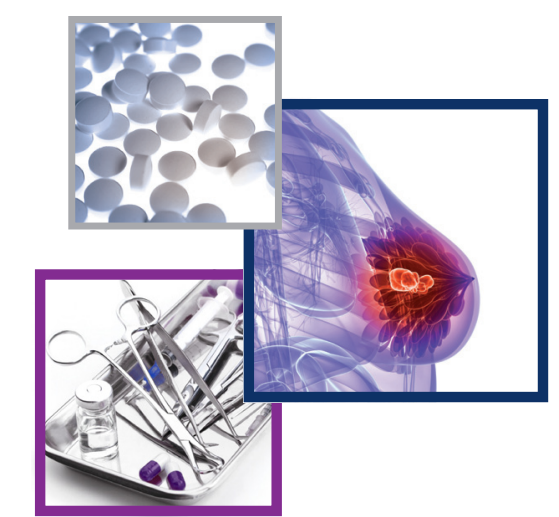

News \& Views

News

Journal Watch

Interview

Conference Scene

*Augmentium Pharma Consulting, Toronto, ON, Canada; george@augmentium.com

Future 
only two things that matter in cancer: does the new drug make my patient live longer or feel better? Those two questions are becoming progressively more difficult to answer in breast cancer, for the reasons that I outlined.

\section{Q What are the most challenging aspects of breast cancer clinical trials at present?}

As I said, first is the issue of demonstrating a survival benefit, which is becoming very difficult, particularly in early lines of therapy. Another problem with survival benefit is related to the fact that the launch prices of many of the new breast cancer drugs are quite high, meaning that payers accross the world are asking for robust survival data before accepting the high drug price. It has now become a catch-22; on the one hand, in the trial, pharmaceutical companies are mandated, often by regulators, to allow patients to crossover once the progressionfree survival end point is reached. However, once the crossover happens, it contaminates the ability to find a survival difference, and yet payers are asking for a survival difference. I am not really sure what the best solution is going forward. We have to put a lot of smart people in a room to try to figure out a solution to this problem.

Second is the question, 'does the drug make patients feel better?'. One of the challenges to date is the way that we measure quality of life. Typically, in randomized trials, we use validated quality-of-life instruments. These are applied at various time points and also at the end, and then we compare the differences between the experimental and control groups. We often find, unfortunately, that there are no significant differences between two drugs in terms of quality of life. Part of the challenge is the way we actually measure quality of life, because as we go forward in a clinical trial, we lose patients. Some patients die, so they cannot complete quality-of-life instruments, and some patients become too ill, so they either leave the study, they do not fill out their questionnaire or they do not come to the clinic. When you have qualityof-life data, you are therefore comparing fit patients in one group versus fit patients in another group. That is why we often do not find significant differences, because we are comparing a subgroup of the original sample, a better-performing, fitter subgroup. Another issue with quality-of-life measurements is as you go forward and lose patients in your trial, you lose considerable statistical power, so even if there is a quality-of-life difference, you have too few patients to be able to identify a significant difference.

\section{Q Do you have any idea of what a} solution to these problems might be?

Especially with breast cancer, we have to get back to sequential trials. Back in the late 1990s there were some really elegant studies that looked at taxanes in combination with anthracyclines versus sequential therapy. The scenario would be doxorubicin plus Taxol versus sequential Taxol then doxorubicin. The findings from those studies were very interesting: the doublets showed a progression-free survival benefit, but there was no difference in overall survival and the doublets were more toxic. The argument is that sequential therapy is as good as doublet therapy with less toxicity. In my opinion, one way to address the challenge with survival benefit would be to study drug A versus drug $B$ followed sequentially by drug $C$ on each arm instead of doing drug $A$ versus drug $B$ and then allowing a crossover to the more active agent upon progression. That way we actually mandate a fixed secondary therapy, so there is no contamination, which would make the survival end points a lot cleaner. There is some precedent to this: in kidney cancer, there are approximately five or six drugs right now in the first, second and third line and they are undergoing the same challenge - they are not able to show survival differences. In a recent meeting, where I was advising a pharmaceutical company on their next trial, we discussed doing a sequential randomized trial as opposed to a doublet trial. Particularly in kidney cancer, you cannot combine these new targeted therapies - they are too toxic - so the only solution is sequential therapy trials.

\section{Q How valuable do you think patient- reported outcomes are when evaluating cancer treatments?}

They are critically important, because at the end of the day, the patients are our customers and we have to make them feel better. Patients put a lot of trust in clinicians to offer them a therapy that will make them feel better and live longer. If we do not ask our customers how the intervention is affecting them, how will we know that it is the best intervention for them? Additionally, payers once again would like to see patient-reported outcomes, because if they are paying a high cost for a drug, they want to be sure that the patients are living longer and feeling better. So patientreported outcomes are critically important. Going back to the quote that my mentor told me, living longer and feeling better is all that counts with new cancer treatments.

However, going back to some of the problems that I highlighted earlier, we are having challenges demonstrating differences in patient-reported outcomes, in particular quality of life, and we need new and smarter ways to address quality-of-life differences. There is one very elegant method that was discussed a few years ago, which has since been used in a few trials. The concept is called palliative response. The way it works is you have a validated quality-of-life scale, say $0-100$, and your outcome is that if patients have an improvement by at least, say, 20 units on the quality-of-life scale at the end of therapy, then they would have achieved a palliative response. Now, this improvement has to be maintained for at least two measurements at least 1 month apart. If a person achieves that end point, they have had a palliative response. This means you can actually measure the proportion of patients between groups that have a palliative response. The nice thing about this measure is that if patients die or are too sick to come to the clinic to fill out the quality-of-life questionnaire, they do not get included in the numerator. In this case, the numerator is the number of patients who have palliative response rates, so it is only those who achieve the palliative responses that get included in the analysis. At the end, sample-size loss does not become an issue, and you are able to compare the proportion of patients between groups who achieve a palliative response, time to achieving a palliative response, duration of palliative response and other things like this. Two years ago, there was a kidney cancer drug 
trial reported at the American Society of Clinical Oncology (ASCO) meeting, with the drug (axitinib) being marketed by Pfizer (NY, USA). In the trial, the secondary end point was a palliative response-type end point. It was called clinical deterioration, which is the opposite to palliative response - it was a 20-point drop in the quality-oflife scale. The study actually measured time to clinical deterioration. What they found with the experimental drug was a reduced rate of patients suffering clinical deterioration. So that was a very elegant way of measuring quality of life with patient-reported outcomes. As I recall, the US FDA did approve the drug and the pharmaceutical company was allowed to put that information on the label that the drug slows clinical deterioration. We are starting to see more of these kinds of measures and I for one am a big advocate of using these alternative patient-reported outcome approaches. This is because, so far, a lot of resources have been put into measuring patient-reported outcomes, but the results have been less than optimal with respect to information for payers and clinicians.

\section{Q How do you see clinical trials changing in the coming years?}

What will likely happen, and we are actually seeing this, is there will be more segmented trials, meaning that patients who are positive for a particular biomarker will be enrolled. This will mean we will have a more alike population. One good example of this is Herceptin ${ }^{\circledR}$ (Genentech, CA, USA). Herceptin was the poster child of what is not really personalized medicine, but segmented or stratified medicine, where we create patient subcategories that are more alike. I foresee a lot more Herceptin-like trials, where there is a validated biomarker and only patients with the biomarker will be randomized for the study. This will increase the likelihood of finding a signal with that particular therapy if it targets a particular pathway in cancer cell growth.

\section{Q What is your opinion on the suggestions for accelerating cancer treatment approval?}

That is a very tough question because, on the one hand, with clinical trials, when a pharmaceutical company goes to the regulator, sample size is no more than approximately 1000-2000 patients, and often that is too small to identify clinically rare but critically important side effects. On the other hand, there are patients who obviously need access to these drugs, so the challenge is do we have enough information about safety and efficacy: does the drug actually work, and is it safe? However, if we do not have enough information and we ask for another trial, this may slow down patient access. Overall, it really is a very difficult negotiation.

\section{Q How do you feel about the} traditional outcomes used in clinical trials? How would you improve on these given the option?

I think the typical outcomes, including response rate, progression-free survival, time to treatment failure and overall survival, will still be used. Hopefully, they will be augmented with better-designed patient-reported outcome end points, so payers and clinicians will have a broad spectrum of information for informed decision-making. I also think we need to track side effects better; for example, many of the side effects from cancer treatment are typically acute events, so longer follow-up post-treatment is important. Once a patient goes home after therapy, follow-up telephone calls can be useful to ask whether there were any toxicities reported or whether the patients have not actually reported something. What can happen in clinical trials and in clinical practice is that patients may be afraid to report certain toxicities in fear that the doctor might stop the therapy or might reduce the dose. One area that I work in is nausea and vomiting research. What we have found in some of our studies is that patients under-report the severity of their nausea and vomiting. We found this out by asking patients whether they were taking nonprescribed therapies at home to treat nausea and vomiting $-20 \%$ of the patients were. With further probing, we found that these patients were having an awful time with nausea and vomiting control, but they did not tell us that, we had to find out indirectly. I think following up with patients and trying to get the true side-effect profile of a new drug is critically important for improving new trials.

\section{Q What does your role as a} pharmaceutical consultant involve? What do you find most interesting about this?

I have a very interesting job. I worked for a public cancer hospital for 8 years, and in 2000, I started my own private consultancy, which is very rewarding and very interesting.

I work primarily in two areas. One is in drug development, so I work with pharmaceutical companies and/or oncologists. The scenario is an oncologist wants to initiate his or her own clinical trial, whether it is a Phase II or III trial, but oncologists are not methodologists or statisticians, they need some assistance in the actual design of the trial, so I help in that aspect. I ask what are the key end points that need to be measured, how should these be measured, what sort of sample size do you need and how should the data be analyzed? I usually have to prepare a statistical analysis plan and all of this has to go into the study protocol. We then approach a pharmaceutical company and ask for funding and access to the drug in order to conduct the study. Then there is the area of data collection, data analysis and writing the final report and the manuscript. I spend approximately half my time doing that.

The second part of my time is spent doing outcomes-based research, which means looking at drugs that have already been approved and are already in clinical practice, and collecting data on how the drug performs in the everyday setting with respect to clinical outcomes, resource utilization and safety. One area that is very popular right now, especially in the USA, is comparative effectiveness analysis. This attempts to use large observational databases to address questions that have not been addressed through clinical trials, and there are many unanswered questions. We use these databases, along with some very interesting 
statistical techniques, to help remove the selection bias that is part of observational databases. We can then test $a$ priori hypotheses. For example, one recent study that I published a few months ago was the impact of Avastin ${ }^{\circledR}$ (Genentech) maintenance therapy in lung cancer. This hypothesis had never been formally tested as a primary objective in a trial. Through an observational database, we found lung cancer patients who received Avastin and matched them to a sample that did not. We measured time to treatment failure, safety and survival, and what we found was that there was indeed a survival benefit in patients who did receive Avastin maintenance therapy. This is the kind of research that I spend the second half of my time doing.

Many of the clients I work with are pharmaceutical companies, governments and also hospitals, so I am able to interact with the three most important stakeholders in the entire cancer treatment process. This means I am able to see different things and hear the different concerns that the three different stakeholders have, which has been very rewarding. It has also been very rewarding working with drugs early on in clinical development. That is often something that I would not have exposure to if I were working at a cancer center. My job also allows me to work in different therapeutic areas, with different clinicians. Working in different areas forces you to keep up with the literature and be on top of your game, because either as a statistician or as an outcomes researcher you get asked different questions from the respective disease sites and you have to know how to respond appropriately - what is the current state of the art for that disease site, and so on.
Q How important do you think cost-effectiveness analyses are for the future of cancer therapies?

If you look over the past 15 years at cancer drug launch prices, there has been an almost exponential increase in the cost of cancer drugs. Drugs are the most easily identifiable expense for payers, who have to cover costs for doctors and hospitals, as well as drugs. However, drug cost is easy to spot and easy to impact - you just do not fund the drug or limit its use. Payers are therefore looking for ways to justify budgetary increases when they go to their respective ministers of health asking for more funding. One way to do that is through cost-effectiveness studies. One example of this is NICE, in the UK. In addition to having an impact in the UK, NICE has a global impact, because many other countries across the world look at NICE decisions and NICE recommendations for their own jurisdiction. In brief, cost-effectiveness studies are incredibly important. I think they are here to stay and will become more important as the next generation of drugs is launched. The drug costs for the most recent launches are in the neighborhood of approximately US\$10,000 ( UK£6000) per month. This means we have to find ways to pay for these drugs and to make effective decisions, and cost-effectiveness analysis is one aspect of the information that is important for making these decisions.

\section{Q Do you think we can ever cure cancer?}

We have certainly had successes with many cancers and there are some cancers now that are curable, for example, testicular cancer and certain lymphomas. Chronic myeloid leukemia is not curable, but it is well controlled with drugs like imatinib. Another statistic is that $50 \%$ of early-stage breast cancer patients are cured, so we have made incredible progress. However, there are some cancers that are a lot more difficult to treat. Cancer cells are very smart - there is an entire evolutionary process behind cancer cells. They can grow in hypoxic environments and they can withstand radiation and chemotherapy. They really are an extraordinary feat of evolution and evolution is very smart. Evolution has had a lot of time to figure things out, and we are only at the tip of the iceberg in our understanding. The progress has really been extraordinary and I am very thankful to be in oncology, because it is such an exciting and extraordinary area of work, especially at this time as we are learning more about cancer cell biology, biomarkers and targeted therapies. In short, I think cancer probably will not be cured in its entirety, but many cancers will certainly be controlled and patients will be able to live for a long time on therapy, but at least they will be able to live with a good quality of life.

\section{Disclaimer}

The opinions expressed in this article are those of the interviewee and do not necessarily reflect the views of Future Medicine Ltd.

Financial \& competing interests disclosure

G Dranitsaris has no relevant affiliations or financial involvement with any organization or entity with a financial interest in or financial conflict with the subject matter or materials discussed in the manuscript. This includes employment, consultancies, honoraria, stock ownership or options, expert testimony, grants or patents received or pending, or royalties.

No writing assistance was utilized in the production of this manuscript. 